\title{
A relação entre a saúde bucal e a cárie dentária em oito comunidades ribeirinhas - Pará, Brasil
}

\author{
The relationship between oral health and dental caries in eight riverside communities - Pará, Brazil
}

\author{
Regiane Cristina do Amaral, ${ }^{1}$ Dirceu Alves Carvalho, ${ }^{2}$ Anthony Brian, ${ }^{1}$ Glaucy Passos Sakai ${ }^{2}$ \\ ${ }^{1}$ Departamento de Saúde Coletiva, Faculdade de Odontologia, Centro Universitário Dr. Leão Sampaio, Juazeiro do Norte, CE, Brasil \\ ${ }^{2}$ Departamento de Gestão Hospitalar, Faculdade de Enfermagem, Centro Universitário UniEvangélico, Anápolis, GO, Brasil \\ - Os autores declaram que não há conflito de interesse.
}

\section{Resumo}

Objetivo: avaliar a prevalência da cárie dentária em crianças/adolescentes de 0 a 15 anos em oito comunidades ribeirinhas do Pará, localizadas as margens do rio Maró, afluente do rio Arapiuns, PA e instituir um programa de promoção de saúde para estas comunidades. Material e Métodos: foi realizado uma visita a cada comunidade, com duração de três dias, sendo no primeiro dia realizado um levantamento epidemiológico em relação à cárie dentária e necessidades de tratamento (critérios da OMS, 1997). No segundo e terceiro dias foram realizadas atividades educativas, preventivas, além de tratamentos odontológicos nesta população. Resultados: foram examinadas 416 crianças/adolescentes na faixa etária de 0 a 15 anos, sendo 183 de 0 a 6 anos e 234 de 7 a 15 anos, sendo 196 do sexo feminino e 218 do sexo masculino. 0 ceod médio para a idade de 5 anos foi de $5,3( \pm 4,5)$ e o CPOD aos 12 anos foi de $4,0( \pm 3,1)$, sendo o elemento predominante 0 cariado. Entre as necessidades de tratamentos mais prevalentes foram restauração de 1 ou 2 faces e extrações em ambas faixas etárias. Observou-se que esta população está sob risco e atividade de cárie dentária, seja pela falta de instrução quanto à higiene bucal e pela dificuldade de acesso à assistência prestada pelo serviço de saúde da região. Conclusão: verificou-se pelo presente estudo que os índices avaliados mostram-se acima dos apresentados nos levantamentos epidemiológicos nacionais (SB Brasil, 2010), demonstrando a necessidade de acesso a serviços odontológicos, bem como mais ações promotoras em saúde a esta população.

Palavras-chave: Saúde bucal; Cárie dentária; Epidemiologia; População indígena.

\section{ABSTRACT}

Objective: to evaluate the prevalence of dental caries among children and adolescents age 0-15 years in eight communities in the state of Pará, located on the banks of the Maró River, a tributary of the Arapiuns River, and to establish a health promotion program for these communities. Material and Methods: a three-day visit to each community was carried out. On the first day, an epidemiological survey was carried out on dental caries and treatment needs (WHO, 1997). On the second and third days, educational and preventive activities were carried out, as well as dental treatments in this population. Results: a total of 416 children/adolescents aged 0 to 15 years were examined; of these 183 were 0-6 years old and 234 were 7-15 years old; 196 were female and 218 were male. The mean DMFT for the age of 5 years was 5.3 ( \pm 4.5 ) and the DMFT at 12 years was 4.0 ( \pm 3.1 ), the predominant element being caries. Among the most prevalent treatment needs were the restoration of 1 or 2 faces and extractions in both age groups. It was observed that this population is under risk, and dental caries activity is either from the lack of education of oral hygiene or from the difficulty of accessing assistance provided from the health services of the region. Conclusion: it was verified by the present study that the indices evaluated are shown to be above those presented in the national epidemiological surveys (SB Brazil, 2010), demonstrating the need for access to dental services, as well as more actions promoting health in this population.

Keywords: Oral health; Dental caries; Epidemiology; Indigenous population.

\section{Introdução}

$\Delta$ s regiões Norte e Nordeste do Brasil possuem características regionais e sociais marcantes, que muitas vezes denotam discrepâncias quando se comparam aos índices de saúde bucal. Afinal, segundo relatório do SB Brasil - 2010, ${ }^{1}$ os valores médios encontrados no índice ceod (dentes cariados, extraídos e obturados), equivalente a idade de 5 anos, se sobressaem negativamente nas regiões Nordeste, Norte e Centro-Oeste, em detrimento as regiões Sudeste e Sul. ${ }^{1}$ Além disso, a representação do componente cariado neste grupo se sobressai em números, enquanto que nas regiões Sul e Sudeste a quantidade de elementos dentários restaurados se mostra significativamente maior. Em relação ao índice CPOD (dentes cariados, perdidos e obturados), estes dados são semelhantes para as idades de 12 anos e 15 a 19 anos. Para a doença periodontal, avaliando crianças aos 12 anos com sextantes hígidos, o menor percentual constatado foi na Região Norte (41,6\%), e o maior na Região Sudeste $(67,9 \%) .{ }^{1}$ Há ainda discrepâncias quando comparado por região, capital e interior, sendo encontradas as piores condições de saúde bucal no interior dos Estados. Isso muito provavelmente devido à dificuldade de acesso aos serviços de saúde, que fazem com que o princípio da "univer- salidade" e "integralidade" do Sistema Único de Saúde (SUS) nem sempre sejam exercidos.

Desde as últimas três décadas do século XX e começo do século XXI, a maioria dos países desenvolvidos conseguiram declinar os índices de cárie dentária. Entretanto, ao se analisar o interior desses países, há diferenças significativas entre as cidades e conjuntos populacionais. ${ }^{2}$

Entre as regiões ou comunidades que têm dificuldade de acesso aos serviços de saúde, estão as comunidades ribeirinhas, que são focos de algumas ações da Política Nacional de Saúde, entre ela a Saúde Bucal, como atitudes de ampliação do acesso, que constituem em atendimento fluvial ou móvel. ${ }^{3}$

Contudo, quando se trabalha com comunidades ribeirinhas, existem alguns fatores que dificultam ainda mais o acesso, como, por exemplo, períodos de cheia ou seca do rio, lugares nos quais grandes barcos, como é a maior parte dos atendimentos fluviais não passam por regiões com rios mais estreitos e não tão profundos, deixando estas comunidades sem qualquer acesso aos serviços. Desta forma, tem-se uma comunidade em que há polarização da doença cárie dentária, entre outras, torna-se mais evidente. ${ }^{4-6}$

Assim, diante do quadro o objetivo do presente estudo foi co- 
nhecer sobre a prevalência da cárie dentária em oito comunidades ribeirinhas e instituir um programa de promoção de saúde para essas comunidades localizadas na região do Alto Arapiuns, Pará, evidenciando a realidade ali presenciada e com isso, contribuindo para futuros planejamentos e ações visando a melhoria da qualidade de vida em saúde destas comunidades.

\section{Material e Métodos}

Participaram do estudo oito comunidades as quais se localizam no Estado do Pará a, aproximadamente, $120 \mathrm{~km}$ a sudoeste da cidade de Santarém, sendo elas Aracati, Fé em Deus, Mariazinha, Nova Canaã, Repartimento, Prainha do Maró, São Francisco do Aruã e Vista Alegre. Todas estas comunidades situam-se em um afluente do rio Arapiuns, o rio Maró, que possui, aproximadamente, 20 comunidades ao seu redor. Assim têm-se nesta região oito comunidades maiores, as quais o estudo foi realizado, devido a estas possuírem melhores estruturas para a realização do projeto de pesquisa, como presença de escolas, igrejas. As crianças e os adolescentes destas comunidades vizinhas eram convidados a participar do projeto e, assim, estas se deslocavam de barco até a comunidade maior.

\section{Características das Comunidades Estudadas}

As oito comunidades estudadas são referência para as demais comunidades, visto que contam com a presença do agente comunitário de saúde (ACS), cuja função principal é alimentar os bancos de dados destas comunidades, registrando casos de nascimentos, óbitos, etc. Estas comunidades possuem como estrutura saneamento básico e escola, no qual é oferecida em algumas delas até o $4^{\circ}$ ano do ensino fundamental, sendo em outras comunidades oferecido até o $8^{\circ}$ ano do ensino fundamental. A energia elétrica utilizada nestas comunidades é proveniente de um gerador de energia, ligado somente durante 4 horas diárias. Para o transporte destas comunidades à cidade mais próxima, Santarém - Pará, tratando de casos de urgência, é realizado via transporte fluvial (sendo cerca de 18 a 20 horas de barco). Com isso, tornase escassos os serviços de saúde a esta comunidade levando em consideração a problemática de acesso e locomoção à estas comunidades. ${ }^{6}$

Esta população em questão tem por base alimentar frutas e animais encontrados na floresta ao redor da comunidade, além disso, plantam mandioca e fabricam farinha. Contudo, as comunidades uma vez por semana, recebem a visita de uma embarcação que vende alguns utensílios e produtos, entre eles gasolina, balas, doces e carne.

\section{Delineamento do Estudo}

O referente estudo foi executado em 2013 e 2014, após aprovação pelo Comitê de Ética em Pesquisa da Faculdade de Odontologia em Piracicaba, sob protocolo do número 007/2013, aprovado em 12/03/2013.

Durante sua realização, cada comunidade foi visitada uma vez, com intervalo de dois meses para cada comunidade a ser visitada. As visitas tiveram durabilidade de três dias, distribuídos da seguinte maneira: o levantamento epidemiológico (exame clínico) realizado em relação à cárie e necessidades de tratamento no primeiro dia, reservando o segundo e terceiro dias ao tratamento odontológico, como também às atividades preventivas (ATF) e educativas em saúde bucal. O exame clínico objetivou avaliar a experiência de cárie dentária, utilizando nesta pesquisa os índices ceo-d e CPOD (índice de dentes decíduos cariados, extraídos e obturados; média de dentes permanentes cariados, perdidos e obturados) e necessidades de tratamento que obedeceram aos códigos e critérios recomendados pela Organização Mundial da Saúde (OMS). ${ }^{7}$

Os exames foram realizados em local com luz natural utilizando-se espátulas de madeira descartáveis. A calibração da equipe, formada por quatro examinadores (cirurgiões-dentistas), foi realizada por meio de discussões teóricas e atividades práticas, simulando as diferentes condições e situações que os profissionais encontrariam durante a realização do trabalho prático. A porcentagem de concordância interexaminadores foi de $95 \%$ para a experiência de cárie (índice CPOD) e a concordância intraexaminadores foi de $98 \%$ a $100 \%$. Os dados foram registrados por anotadores previamente treinados, em fichas individuais, cujo modelo simplificado foi proposto pela OMS, $1997 .^{7}$

A amostra não probabilística foi compreendida por 416 crianças/adolescentes, na faixa etária de 0 a 15 anos, das comunidades ribeirinhas, que compareceram no dia da avaliação e que apresentaram o consentimento pelos pais/responsáveis, por meio da assinatura do Termo de Consentimento Livre e Esclarecido - protocolo 007/2013. Todas as crianças e adolescentes presentes nas comunidades no dia do estudo foram incluídos e examinados, exceto as crianças que não tinham dentes erupcionados.

Durante o segundo dia de pesquisa, foram entregues aos participantes os kits contendo dentifrícios fluoretados, escovas e fio dental, além de um copo, utilizado para individualizar cada kit de higiene oral. Foram ainda realizadas instruções sobre a importância destes kits serem guardados de forma individual, devendo manter as demais escovas dentais de familiares guardadas sem aglomeração para evitar contaminação cruzada.

Realizou-se ainda escovação supervisionada e acompanhada com os devidos pais e responsáveis de cada criança, com aplicação tópica de flúor fosfato acidulado (ATF), correspondente com o eventual risco de cárie dentária.

Os pais e/ou responsáveis participaram efetivamente em todas as atividades desenvolvidas, assegurando a confiabilidade dos mesmos perante a equipe. Os procedimentos e tratamentos odontológicos foram feitos por quatro cirurgiões-dentistas, considerando a necessidade encontrada. A educação em saúde foi enfatizada de forma significativa, dando prioridade à técnica restauradora atraumática (TRA), tratamentos não invasivos, uma vez que a maioria das pessoas que ali residem nunca foram ao dentista, mudando o paradigma do acesso ao serviço odontológico vinculado apenas a extração dentária aliada à dor. As crianças e adolescentes participantes da pesquisa tiveram ali seu primeiro contar com o cirurgião-dentista, exceto poucos examinados que buscaram atendimento odontológico na cidade de Santarém, a fim de realizar exodontias (figuras 1, 2 e 3). Os dados obtidos nesta pesquisa foram digitados numa planilha Excel e realização das análises descritivas. 

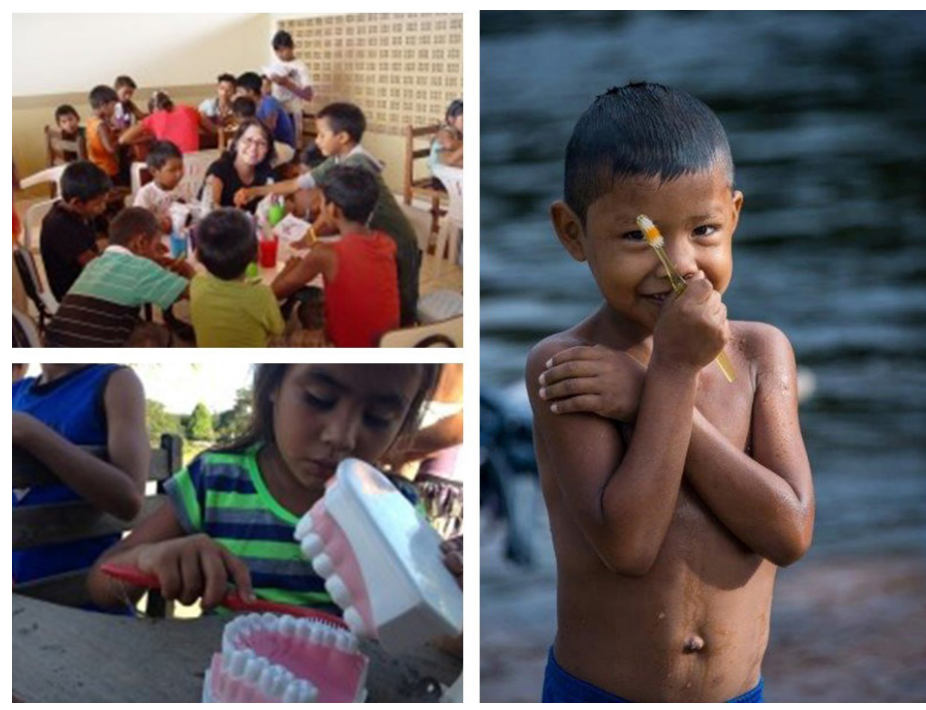

Figura 1. Atividades de educação em saúde, Pará - 2013/2014
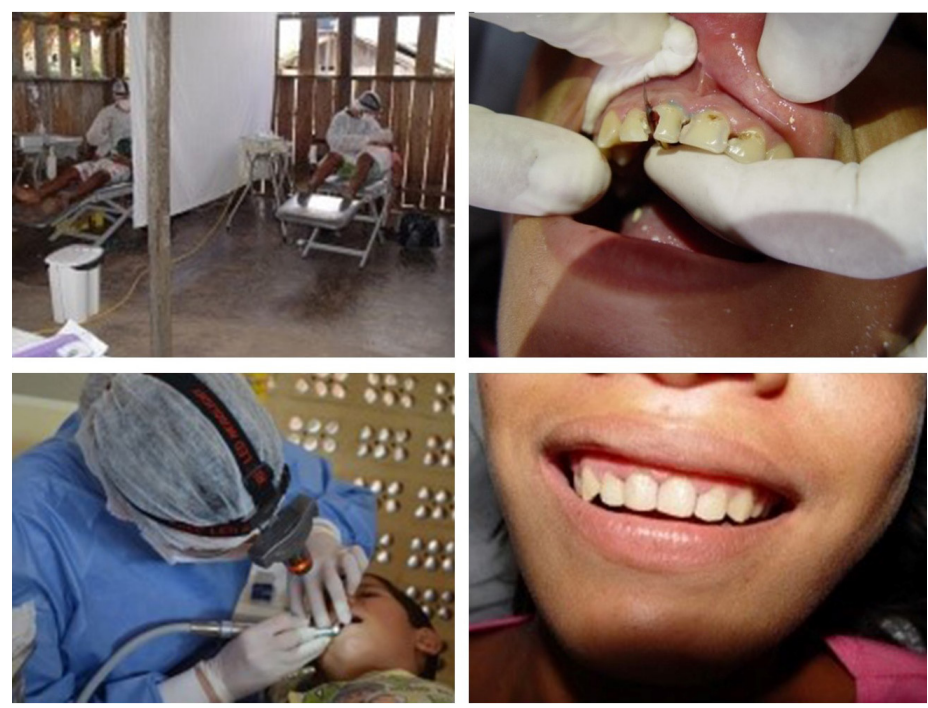

Figura 2. Atendimentos clínicos realizados, Pará 2013/2014

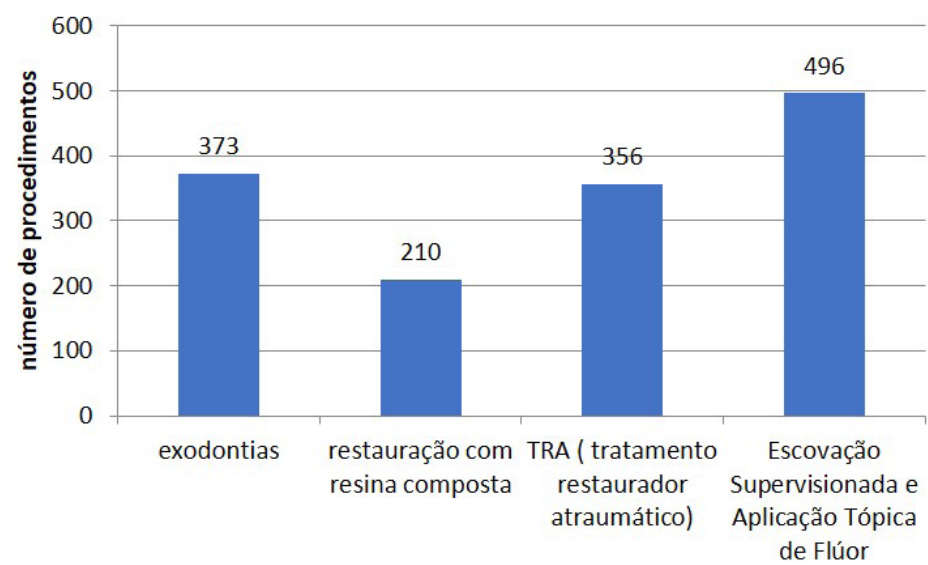

Figura 3. Procedimentos clínicos realizados, Pará 2013/2014

\section{Resultados}

Foram examinadas 416 crianças/adolescentes na faixa etária de 0 a 15 anos, sendo 183 de 0 a 6 anos e 234 de 7 a 15 anos, sendo 196 do sexo feminino e 218 do sexo masculino. O ceod médio para 5 anos foi de $5,3( \pm 4,5 ; \mathrm{n}=30$, sendo $86 \%$ cariado e $13 \%$ extraído) e o CPOD aos 12 anos foi de $4,0( \pm 3,1 ; \mathrm{n}=28$, sendo $85 \%$ cariado e $15 \%$ extraído).

Entre os tratamentos mais prevalentes (segundo classificação da OMS7) tem se os casos de ATF e escovação supervisionada, seguido de restauração de uma ou duas faces e extrações em ambas faixas etárias, sendo encontrados indicações de restauração de uma superfície para 241 dentes decíduos e 416 dentes permanentes, restauração de duas ou mais superfícies para 230 dentes decíduos e 306 dentes permanentes e extrações indicadas para 110 dentes decíduos e 230 dentes permanentes.

Previamente ao atendimento clínico, todos os pacientes foram submetidos a bochechos e profilaxia com gaze da cavidade oral com digluconato de clorexidina $0,12 \%$.

Assim, entre os tratamentos odontológicos realizados o que houve maior prevalência foram ATF e escovação supervisionada, seguido de exodontias. Foram realizadas também restaurações em resina composta.

Entre os tratamentos curativos realizados observa-se grande número de restaurações do tipo TRA, algumas utilizando o ionômero de vidro convencional e outras o ionômero de vidro resinoso, realizados tanto em dentes decíduos como permanentes devido à rapidez do procedimento e indicação, como população considerada de alto risco para cárie (população não exposta a fluoreto de forma sistêmica, falta de acesso a serviços de saúde, Tabela 1).

Tabela 1. Número de crianças e adolescentes examinados de acordo com a idade e índice ceod/CPOD médio - Pará, 2013/2014

\begin{tabular}{c|c|c}
\hline Idade & Examinados & ceod /CPOD \\
\hline 1 & 31 & 0,22 \\
\hline 2 & 23 & 2,56 \\
\hline 3 & 28 & 2,35 \\
\hline 4 & 40 & 3,55 \\
\hline 5 & 30 & 5,3 \\
\hline 6 & 31 & 5,1 \\
\hline 7 & 23 & $0,52^{*}$ \\
\hline 8 & 46 & $1,54^{*}$ \\
\hline 9 & 25 & $1,24^{*}$ \\
\hline 10 & 36 & $2 *$ \\
\hline 11 & 21 & $2,33^{*}$ \\
\hline 12 & 28 & 4 \\
\hline 13 & 16 & 4,43 \\
\hline 14 & 17 & 4,2 \\
\hline 15 & 21 & 5,57 \\
\hline
\end{tabular}

*CPOD - dentes permanentes cariados, perdidos e obturados 


\section{Discussão}

A cárie dentária, apesar de ainda ser a doença mais prevalente na cavidade bucal, ${ }^{8}$ se apresenta de forma desigual entre as regiões brasileiras. No Brasil, verificamos que houve um decréscimo ao avaliarmos o índice CPOD para a idade de 12 anos, visto que na década de 80 este era em torno de 7,6, em 1993 de 4,8, em 2003 de 2,8 e em 2010 igual a 2,07. Na região Norte (idade de 12 anos), verificou-se que a doença declinou de 7,50 (em 1986) para 3,13 (em 2003), e manteve-se em 3,16 (em 2010), apresentando a maior prevalência de cárie em todo o Brasil por região., ${ }^{1,2}$

Ao se avaliar ainda o interior da região Norte, podemos observar que este índice pode chegar até a 3,4. No presente estudo ao se verificar os índices de saúde bucal (CPOD) em oito comunidades ribeirinhas na região Norte do país, foi possível verificar que o índice CPOD (12 anos) encontrado foi superior à média da região Norte interior $(4,0 \pm 3,1 ; n=28$, sendo $85 \%$ cariado e $15 \%$ extraído).

Segundo dados do SB Brasil 2010, a média de ceod aos 05 anos na Região Norte interior é de 3,37, sendo no presente estudo encontrado valor superior aos dados nacionais $(5,3( \pm 4,5 ; \mathrm{n}=30$, sendo $86 \%$ cariado e $13 \%$ extraído).

Dados semelhantes ao do presente estudo foram encontrados em outros trabalhos que envolvem comunidades ribeirinhas, os quais alegam a necessidade de políticas preventivas de saúde pública, ofertando também mais acesso ao atendimento odontológico a regiões de difícil acesso. A elaboração de pesquisas e programas de saúde com base nas características regionais reduziriam as grandes desigualdades sociais existentes devido à ausência de políticas públicas direcionadas as populações rurais, em especial o ribeirinho. ${ }^{9,12}$

No estudo realizado por Silva et al., ${ }^{4}$ os autores encontraram em população ribeirinha residente às margens dos rios Machado e Preto (Rondônia, Brasil), em 2005 e 2006, na faixa etária de 4-5 anos de idade, ceod $=4,30 ; 6-10$ anos, $\mathrm{CPOD}=1,04$, ceod $=3,52$; aos 12 anos, $\mathrm{CPOD}=2,65$. No estudo realizado por Nascimento e Scabar, ${ }^{10}$ realizado com indígenas, verificou-se que o ceod aos 5 anos encontrado foi de 3,5 diferente do presente estudo, contudo similares em prevalência do elemento cariado.

Evidenciam-se assim as necessidades discrepantes em relação à saúde bucal ao se comparar as diferentes regiões do país e ainda mais ao se equivaler as regiões interioranas do Estado, não sendo raros casos em que em apenas uma criança se é possível realizar até 10 TRA (tratamento restaurador atraumático), ou seja, crianças com até 10 dentes acometidos pela doença cárie dentária.

Nas comunidades visitadas no presente estudo, verifica-se a existência de projetos locais, como o barco ABARE, ${ }^{3}$ na tentativa de se ampliar a atenção em saúde para estas populações, como preconizado pela Política Nacional de Saúde Bucal. ${ }^{13}$ Contudo, devido a processos de cheias e secas dos rios ou ainda por a se tratar de um rio afluente, no qual as oito comunidades estão alocadas, as embarcações que mantém assistência médica e odontológica não conseguem alcançar estas comunidades do presente estudo.

A alimentação dos ribeirinhos, semelhante aos dos povos indígenas, como em muitos casos há uma descendência direta, baseia- se em beiju, tapioca, farinha amarela e sucos de frutas. Contudo, como o rio ao qual se localizam tais comunidades é considerado o rio da fome, que devido a particularidades da água, esta região é pobre em peixes, os ribeirinhos deste local se alimentam de caças como anta, paca, macaco, tatu, veado, pássaros, jacaré, entre outros.

Diferente de povos indígenas, os ribeirinhos no presente estudo têm hábitos de dieta influenciados pela cidade mais próxima, ou seja, Santarém - PA, pois uma embarcação passa pelas comunidades semanalmente oferecendo produtos, entre os quais se incluem balas, guloseimas e açúcar, um fator agravante para a doença cárie dentária. ${ }^{9}$

O presente estudo buscou, além de conhecer a saúde bucal da população ( 0 a 15 anos) em relação à cárie dentária, realizar atividades educativas e preventivas em saúde bucal.

Assim, no período em que a equipe de saúde bucal esteve presente nas comunidades e quando retornava entre as visitas seja para reposição de escovas dentais (a cada 2 a 3 meses) ou conversas com os líderes das comunidades e professores observou-se que a higiene bucal foi incluída no dia a dia da comunidade. Mudanças de hábito foram observadas nas visitas da equipe a comunidade, em que era possível ver que as crianças escovam os dentes durante o banho no rio que ocorre basicamente duas vezes ao dia, um pela manhã e outro no final da tarde (figura 1) e nenhum caso de falta de aula na escola por dor foi notificado.

Nas comunidades visitadas têm se ainda a presença de escolas com professores que moram na comunidade durante o período letivo e nas férias voltam para suas cidades de origem, no processo de educação em saúde, tais professores eram envolvidos no processo e verificou-se que o hábito de escovação dentária foi introduzido nas atividades escolares, ou seja, após a merenda escolar as crianças escovavam seus dentes.

Certamente que a educação em saúde é um processo continuo e em comunidades remotas torna-se uma tarefa ainda mais desafiadora. Contudo, diante deste desafio as metas de uma saúde bucal com compromisso com a saúde e o bem-estar do próximo envolvido na profissão da Odontologia torna-se algo plausível de serem realizados.

\section{Conclusão}

Verifica-se pelo presente estudo que os índices avaliados mostram-se maiores que os apresentados nos levantamentos epidemiológicos nacionais, ${ }^{1}$ demonstrando a necessidade de acesso a serviços odontológicos a esta população, além de continuidade de programas preventivos e educativos na região. Foram observadas ainda pequenas mudanças de hábito como escovação dentária durante os banhos nos rios e no intervalo após as refeições na escola.

\section{Agradecimentos}

Ao comandante Lelis Fachini Filho, organizador do projeto saúde bucal - Alto Arapiuns. Aos dentistas Junio Chaves Queiroz, Joel de Morais Tavares, Anderson de Andrade Doutor e Rochelli C G Silva Passos que participaram do projeto. A empresa TAM por financiar parte do projeto. 


\section{Referências}

1 - BRASIL. Ministério da Saúde. SB Brasil 2010: Resultados Principais [internet]: 2012 [citado em 2014 mai 4]. Disponível em: http://189.28.128.100/dab/docs/geral/projeto_sb2010_relatorio_final.pdf

2- Narvai PC, Frazão P, Roncalli AG, Antunes JLF. Cárie dentária no Brasil: declínio, iniquidade e exclusão social. Rev Panam Salud Publica. 2006;19(6):385-93. 3- Brasil. Ministério da Saúde [internet]: 2013. [citado 2016 fev 2]. Disponível em:http://www.brasil.gov.br/saude/2013/10/ministerio-da-saudeampliarecursos-para-ubs-fluvial-abare-i

4 - Silva RHA, Castro RFM, Cunha DCS, Almeida CT, Bastos JR, Camargo LMA. Cárie dentária em população ribeirinha do Estado de Rondônia, Região Amazônica, Brasil, 2005/2006. Cad Saúde Pública. 2008;24(10):2347-53.

5 - Bezerra ESM, Nogueira AJS. Prevalência de Perdas Dentárias Precoces em Crianças de População Ribeirinha da Região Amazônica. Pesq Bras Odontoped Clin Integr.2012;12(1):93-8.

6- Carvalho DC, Passos GSL, Amaral RC. Prevalência de cárie e necessidades de tratamento em duas comunidades ribeirinhas - Pará, Brasil. J Health Sci Inst. 2014;32(1):23-7.

7 - WHO. World Health Organization. Oral health surveys, basic methods. 4nd ed. Geneve:WHO;1997.

8 - WHO. World Health Organization. The World Oral Health report [internet]:
2010.[ citado em 2016 mai 10]. Disponível em:http://www.who.int/mediacentre/ factsheets/fs318/en/index.html.

9 - Programa Alto Arapiuns de Desenvolvimento Sustentável. Histórico [internet]: 2015 [citado em 2016 jun 06]. Disponível em: http://webcache.googleusercontent.com/search?q=cache:5bLMKNllUkYJ:altoarapiuns.com.br/historico $/+\& c d=1 \& h l=p t-B R \& c t=c \operatorname{lnk} \& g l=b r$

10 - Nascimento S, Scabar LF. Levantamento epidemiológico de cárie, utilizando os índices CPO-D, ceo-d e IHOS, nos índios da aldeia Wakri no Estado do Pará. J Health Sci. 2008;26(2):246-53.

11- Cohen-Carneiro F, Souza-Santos R, Pontes DG, Salino AV, Rebelo MAB. Oferta e utilização de serviços de saúde bucal no Amazonas, Brasil: estudo de caso em população ribeirinha do Município de Coari. Cad Saúde Pública. 2009;25(8):1827-38.

12 - Frazão P, Santos CRI, Benicio DEA, Marques RAA, Benício MHA, Cardoso MA, et al. Cárie dentária em escolares de 12 anos de idade em município sem água fluoretada na Amazônia Ocidental brasileira, 2010. Epidemiol Serv Saúde. 2016;25(1):149-58.

13 - BRASIL. Ministério da Saúde.Política Nacional de Saúde Bucal [internet]:2015 [citado em 2016 jul 05]. Disponível em: http://dab.saude.gov.br/portaldab/pnsb.php

\section{Mini Currículo e Contribuição dos Autores}

1. Regiane Cristina do Amaral - cirurgiã-dentista e mestre. Contribuição: contribuição científica e intelectual.

2. Dirceu Alves Carvalho - cirurgião-dentista. Contribuição: aquisição dos dados.

3. Anthony Brian - graduando de Odontologia. Contribuição: interpretação dos dados.

4. Glaucy Passos Sakai - enfermeira. Contribuição: aquisição dos dados.

Recebido em: 03/10/2016 / Aprovado em: 19/01/2017

Autor Correspondente

Regiane Cristina do Amaral

E-mail: amaralre@yahoo.com.br 